\title{
Osteoid osteoma of the femur in a 7-month-old infant treated with radiofrequency ablation
}

\author{
Warapat Virayavanich • Ravi Singh • \\ Richard J. O'Donnell • Andrew E. Horvai • \\ Robert E. Goldsby • Thomas M. Link
}

Received: 2 April 2010 /Revised: 13 July 2010 /Accepted: 22 July 2010 /Published online: 8 August 2010

(C) The Author(s) 2010. This article is published with open access at Springerlink.com

\begin{abstract}
Osteoid osteoma occurs most commonly in children, adolescents, and young adults between the ages of 5 and 30 years. In the preschool age group, it is quite uncommon, accounting for only $3-8 \%$ of all osteoid osteoma cases. We report a case of osteoid osteoma in a 7-month-old infant, who presented with decreased use of the right lower extremity due to pain. Magnetic resonance imaging (MRI) showed an atypical appearance. A biopsy of the lesion, with histopathological examination, confirmed the diagnosis of osteoid osteoma. Radiofrequency ablation (RFA) of the nidus under computed tomography (CT) guidance was performed. The patient developed a recurrence after 3 months, which was treated with a second RFA. On subsequent follow-up, the infant did not show signs of pain after 1 month. In summary, this case report shows that osteoid
\end{abstract}

W. Virayavanich $\cdot$ R. Singh · T. M. Link

Department of Radiology, University of California San Francisco, San Francisco, CA, USA

R. J. O’Donnell

Department of Orthopaedic Surgery,

University of California San Francisco,

San Francisco, CA, USA

A. E. Horvai

Department of Pathology, University of California San Francisco, San Francisco, CA, USA

R. E. Goldsby

Department of Pediatrics, University of California San Francisco, San Francisco, CA, USA

T. M. Link $(\varangle)$

Department of Radiology and Biomedical Imaging,

University of California San Francisco,

400 Parnassus Ave., A 367, Box 0628, San Francisco, CA 94143-0628, USA

e-mail: tmlink@radiology.ucsf.edu osteoma can present in early infancy and can be successfully treated with RFA at this age, however, recurrence after the procedure can occur and close follow-up is recommended.

Keywords Infant - Osteoid osteoma - Radiofrequency ablation $\cdot$ Femur

\section{Introduction}

Osteoid osteoma (OO) is a relatively common benign skeletal lesion. It is the third most common primary benign skeletal neoplasm, accounting for approximately $10-12 \%$ of benign bone tumors [1]. Although reported in almost every bone, it most commonly occurs in the long bones of the lower extremity, with $50 \%$ of the cases in the tibia and femur $[2,3]$. Male patients are affected more than female patients at a ratio of 2-3:1 [1-4]. Osteoid osteoma is uncommonly seen in patients before age 5 years or after age 30 years, although a range from 8 months to 70 years has been reported $[2,5]$.

Most patients present with pain, which worsens at night and is relieved by nonsteroidal anti-inflammatory drugs (NSAIDs). The characteristic radiographic findings of $\mathrm{OO}$ include a cortical based central nidus of vascular osteoid tissue surrounded by reactive sclerotic bone [1]. The clinical and radiological picture may be characteristic, but occasionally it is unclear, contributing to delay in diagnosis, especially in a young patient who may not be able to communicate his or her symptoms. Although there are reports of $\mathrm{OO}$ undergoing spontaneous regression, early diagnosis enables timely treatment, avoids unnecessary suffering, and minimizes morbidity including bone deformity and leg-length discrepancy [6]. We report a 7-month-old female infant with osteoid osteoma, who presented with 


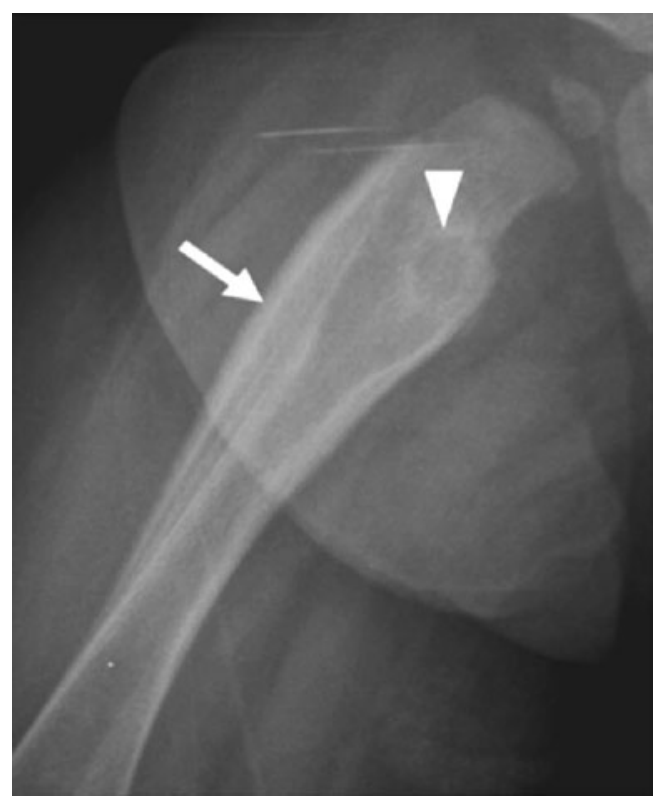

Fig. 1 Roentgenogram of the right hip shows a lytic lesion with sclerotic rim centered in the intertrochanteric region of the proximal right femur (arrowhead). There is lamellar periosteal reaction involving the proximal femur extending to the mid diaphysis (arrow)

limited use of the right lower extremity and exhibited atypical imaging findings. After biopsy tissue confirmation of the diagnosis of OO, she was subsequently treated with radiofrequency ablation (RFA). The patient demonstrated signs of recurrent pain 3 months after the initial procedure and was treated successfully with a second RFA.

\section{Case report}

A 7-month-old girl was referred to our institution in December 2009 with a right femoral lesion, with concerns of neoplasm or osteomyelitis. Her parents noticed that she had shown a decrease in the use of her right lower extremity especially at about 3 months of life when the patient would not bear weight on her right lower extremity when held up in an upright position. The patient's ability to sit upright was also somewhat limited because she preferred to have her right hip and knee extended. At age 7 months, her pediatrician obtained a roentgenogram that showed a lytic lesion in the right proximal femur. The past medical history was unremarkable. Clinical examination showed an afebrile infant who moved her right lower extremity much less than the left and preferred keeping her right hip and right knee extended with external rotation. There was no erythema, warmth or mass in the right lower extremity. The results of routine laboratory studies were normal.

Roentgenograms of the right femur showed an osteolytic area in the intertrochanteric region, surrounded by a sclerotic rim and lamellar periosteal reaction (Fig. 1). A magnetic resonance imaging (MRI) study showed a focal, $1.1 \times 0.9 \times 1.2 \mathrm{~cm}$, high T2 signal lesion centered in the intertrochanteric region of the proximal right femur. There was marked lamellar periosteal reaction involving the proximal right femoral metaphysis extending to the mid diaphysis, with extensive, ill-defined intra- and extraosseous edema (Fig. 2). The differential diagnosis included chronic osteomyelitis with a Brodie's abscess, eosinophilic granuloma, osteoid osteoma, and small round blue cell tumor such as Ewing sarcoma or metastatic neuroblastoma.

A CT-guided core needle biopsy of the lesion was performed. The axial CT scan at the time of biopsy showed a 1-cm lucent lesion eccentrically located within the medial aspect of the proximal femur with marked periosteal new bone formation (Fig. 3). Cultures of the biopsy tissue were negative for fungal and bacterial organisms. Histological examination of the biopsy tissue showed an area of new woven bone formation with prominent osteoblastic rimming in a background of loose, cellular, fibrous stroma (Fig. 4). There were no features of acute osteomyelitis, small round blue cell tumor, or eosinophilic granuloma. A diagnosis of OO was considered.

The infant subsequently underwent an RFA procedure, performed under CT guidance and general anesthesia. To reduce radiation exposure, only $20 \mathrm{mAs}$ with $80 \mathrm{kVp}$ was used,
Fig. 2 Axial T1-weighted pre-contrast (a) and fat-saturated post-contrast (b) MR images of the right femur show a tumor nidus (arrows) associated with extensive bone marrow edema pattern and extra-osseous edema pattern involving the proximal metadiaphysis and diaphysis
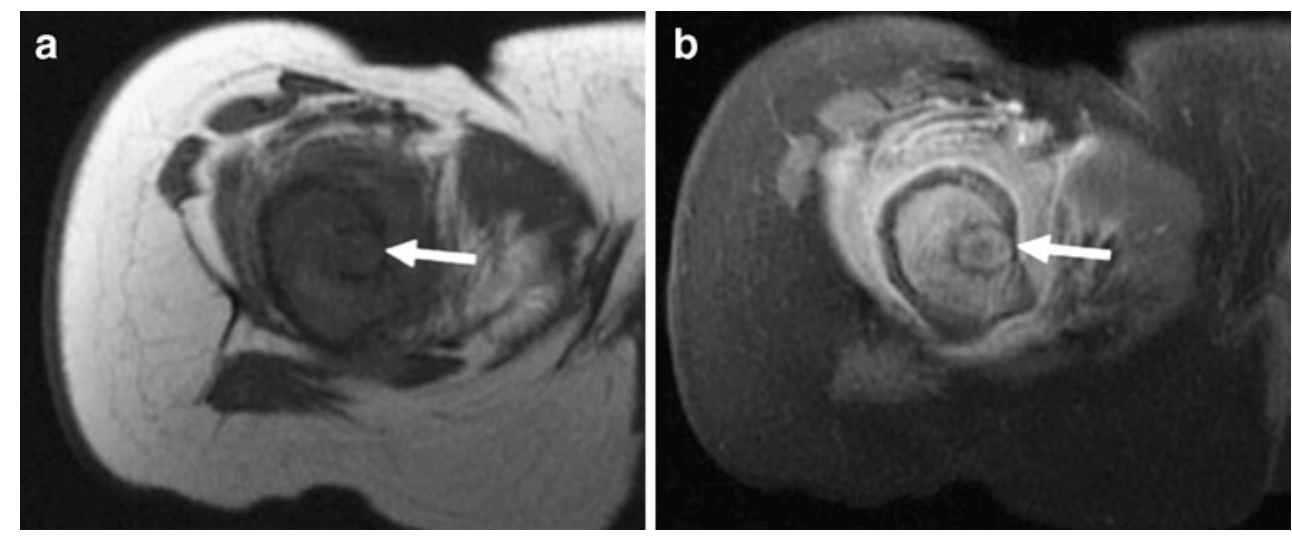


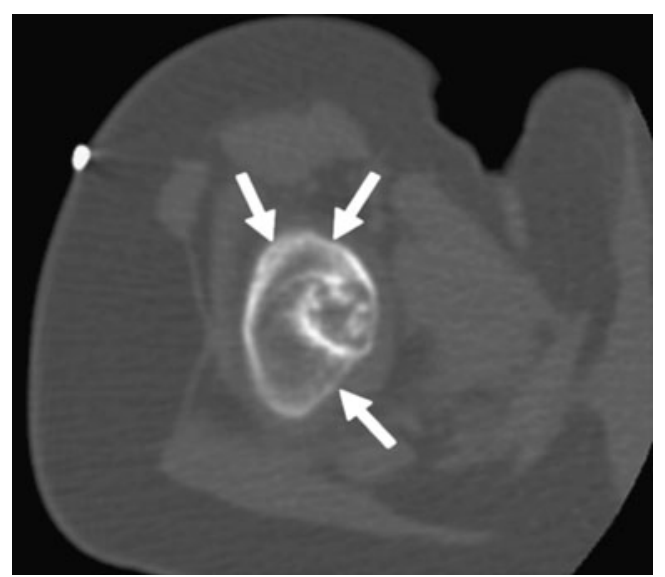

Fig. 3 Axial CT scan (bone window) during percutaneous RFA shows a radiolucent intramedullary nidus with central calcification surrounded by a dense rim of sclerosis. There is a marked periosteal reaction around the cortex (arrows). Note a marker for planning the skin entry point

which resulted in markedly increased image noise, however, still allowing visualization of the lesion. Cefazolin sodium was administered during the procedure as a prophylactic to avoid osteomyelitis. After placement of the electrode-grounding pads and marking the entrance site on the skin, a 14G Bonopty penetration set (AprioMed, Uppsala, Sweden) was inserted and attached to the cortical bone. Then a $16 \mathrm{G}$ drill and extended drill (AprioMed) were advanced to the lesion.

Subsequently, RFA was performed using a Cool-tip ${ }^{\mathrm{TM}}$ RFA system (Tyco Valleylab, Boulder, CO) in a noncooled mode. A $17 \mathrm{G} 15 \mathrm{~cm}$ RFA probe with a $0.7 \mathrm{~cm}$ active tip was inserted. Using this probe and a standard heating procedure by heating the tip of the electrode to $90^{\circ} \mathrm{C}$ for a period of $6 \mathrm{~min}$, a tissue area of $0.5 \mathrm{~cm}$ around the active

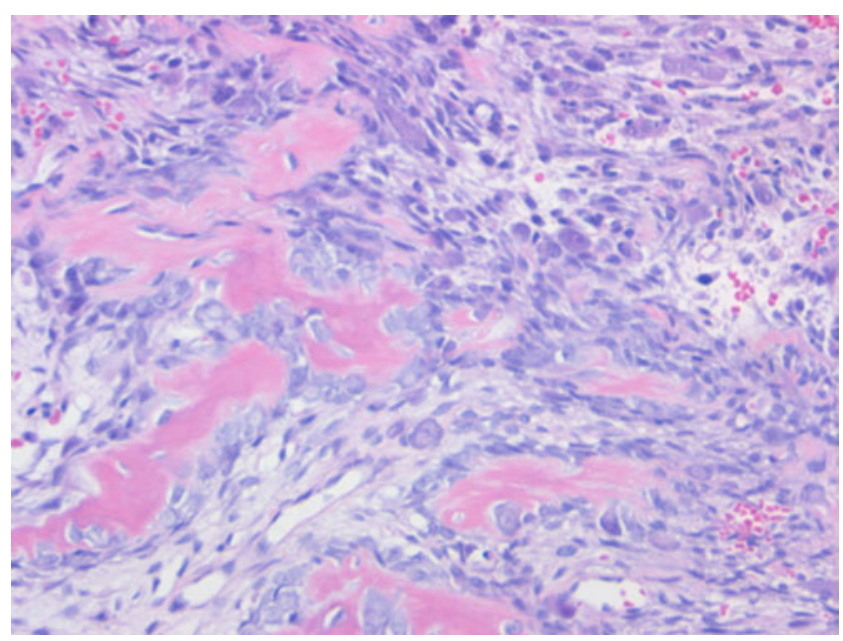

Fig. 4 The tumor consisted of delicate trabeculae of woven bone with osteoblastic rimming. The intertrabecular space was occupied by a loose, hypocellular, vascular stroma. (Hematoxylin and eosin stain, $400 \times$ original magnification) tip was ablated. No complications were observed during or immediately after the procedure. The patient was discharged the next day.

At the follow-up 1 week later, the patient was able to place weight on her right leg when held up in an upright position and sit, unassisted, for several minutes. The patient presented again 3 months later with signs of recurrence, including inability to bear weight and decrease in the use of her right lower extremity. The follow-up radiographs showed persistent osteolytic area in the intertrochanteric region but less prominent periosteal reaction as compared to the radiographs prior to treatment. A second CT-guided RFA was performed 4 months after the initial procedure by using again a $0.7 \mathrm{~cm}$ active tip RFA probe with the same standard technique. The CT images showed a decrease in size of the nidus, which measured $0.7 \mathrm{~cm}$ in diameter (Fig. 5). Additionally, the periosteal reaction has improved when compared to the initial scans. One month after the second ablation, the patient still did not show signs of pain and began to stand on her legs.

\section{Discussion}

There are numerous reports in the literature of osteoid osteomas occurring in patients younger than 5 years of age, but only rarely in those under 1 year of age $[2,5,7,8]$. Habermann and Stern [5] reported a case of OO in an 8-month-old infant whose onset of symptoms began at 4 months. A case of possible congenital OO involving a distal phalanx of the left long finger was reported by Szabo and Smith [9]. That patient was diagnosed when she was 15 years old. However, deformity of the affected phalanx and its nail apparently had existed from birth and gone unnoticed.

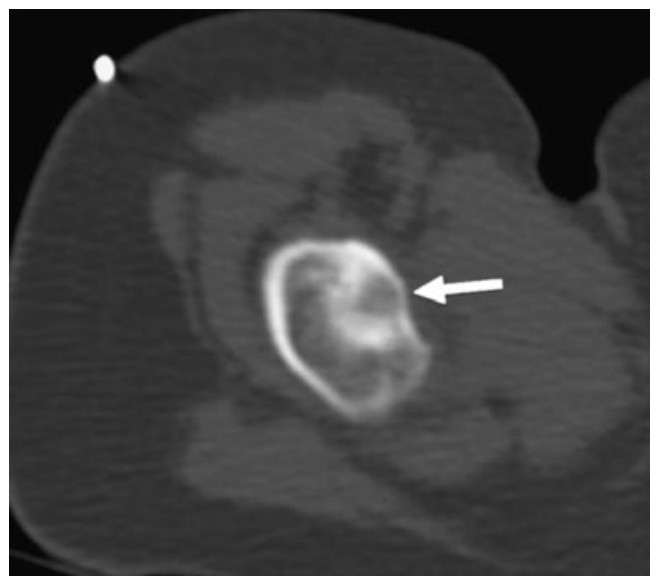

Fig. 5 Axial CT scan (bone window) obtained during the RFA of the recurrent OO. Note mild decrease in size of the nidus (arrow) and periosteal bone formation 
Pain is the most common clinical manifestation of OO, with limp, tenderness, swelling, and atrophy the next most frequent findings in children [7, 8]. The symptoms may be present for periods ranging from weeks to several years before diagnosis [2]. Although the clinical features of $\mathrm{OO}$ in children are similar to those in adults, greater clinical discernment is required for diagnosis, as children might not be able to accurately communicate their symptoms. In our patient, the most prominent sign was decreased use of the affected leg. Although this was first noted by the parents shortly after birth, the appropriate radiologic evaluation of the lesion was delayed until other indications of disease became evident.

The most characteristic and most common radiologic finding of $\mathrm{OO}$ includes a radiolucent, intracortical nidus, containing a variable amount of mineralization, surrounded by an area of dense fusiform reactive sclerosis $[1,10]$. Approximately $20 \%$ of osteoid osteomas involve the medulla. The intramedullary lesions are often barely visible and usually induce only mild or no reactive bone formation [11]; diagnosis of these osteoid osteomas may be challenging as shown by our case.

The nidus of an osteoid osteoma rarely exceeds $1.5 \mathrm{~cm}$ in the greatest dimension. The reactive periosteal bone formation is usually solid. CT scan is generally regarded as the preferred cross-sectional technique to locate the lesion. In children, an MRI study is often performed first in order to limit radiation exposure. The tumor nidus, however, may be difficult to identify on MR imaging [12]. The great sensitivity of MR imaging to detect nonspecific, reactive changes in the bone marrow and soft tissue may result in a misleading aggressive appearance [13]. In our patient, intramedullary location, extensive bone marrow, and soft tissue edema adjacent to the lesion, as well as lamellar periosteal new bone formation, made the diagnosis more difficult; because of this imaging appearance and the unusual age of our patient, other entities, such as osteomyelitis and aggressive tumors, were included in the differential radiologic diagnosis.

Osteoid osteoma and osteoblastoma are considered as closely related processes. Histologically, osteoid osteoma is similar to osteoblastoma and the difference between these two entities is established on the basis of the size of the nidus ( $>2 \mathrm{~cm}$ is generally considered as osteoblastoma), skeletal distribution, radiographic appearance, and clinical presentation [14].

A rare complication of osteoid osteoma is localized overgrowth and deformity of bone. It has been found most frequently in children with symptom onset before 5 years of age, emphasizing the importance of prompt definitive treatment to prevent leg length discrepancy and deformity in infants and very young children [15]. Radiofrequency ablation for the treatment of OO was first described in 1992
[16], and is currently the therapeutic treatment of choice for $\mathrm{OO}$ in both adult and pediatric cases; it is minimally invasive, safe and has a high technical and clinical success rate [17-19]. Successful treatment of OO with RFA was reported in a 1-year-old boy by Egstrom et al. [20], but reviewing international medical literature our patient was the youngest child with OO to have been treated with RFA. In some studies, a higher recurrence of osteoid osteoma following treatment with RFA has been suggested in children or younger patients $[17,21]$ and association of recurrence with large nidus diameter $(1.0-1.5 \mathrm{~cm})$ has also been reported $[18,22]$. Our case demonstrates potential problems with RFA in very young patients with relatively large lesions $(1.2 \mathrm{~cm}$ in maximum diameter of the nidus). Although our patient developed a recurrence after the primary treatment, with a short follow-up after the second ablation, she is currently doing well. Whether there are any long-term consequences of the $\mathrm{OO}$ or the RFA procedure remains to be seen.

In summary, osteoid osteoma can be a diagnostic challenge when it presents with atypical clinical features in a very young patient. But also in very young patients, RFA is a safe procedure and should be considered as the treatment of choice. However, recurrence after the procedure is more frequently observed and close follow-up is recommended.

Acknowledgments We would like to thank Dr. Daniel I. Rosenthal, Department of Radiology, Massachusetts General Hospital for his kind advice and support with the management of this patient.

Open Access This article is distributed under the terms of the Creative Commons Attribution Noncommercial License which permits any noncommercial use, distribution, and reproduction in any medium, provided the original author(s) and source are credited.

\section{References}

1. Dorfman HD, Czerniak B. Benign osteoblastic tumors. In: Dorfman HD, Czerniak B, editors. Bone tumors. St. Louis: Mosby; 1998. p. 85-127.

2. Cohen MD, Harrington TM, Ginsburg WW. Osteoid osteoma: 95 cases and a review of the literature. Semin Arthritis Rheum. $1983 ; 12: 265-81$

3. Jackson RP, Reckling FW, Mants FA. Osteoid osteoma and osteoblastoma. Similar histologic lesions with different natural histories. Clin Orthop Relat Res. 1977;128:303-13.

4. Schajowicz F, Lemos C. Osteoid osteoma and osteoblastoma. Closely related entities of osteoblastic derivation. Acta Orthop Scand. 1970;41:272-91.

5. Habermann ET, Stern RE. Osteoid-osteoma of the tibia in an eightmonth-old boy. A case report. J Bone Joint Surg Am. 1974;56:633-6.

6. Kneisl JS, Simon MA. Medical management compared with operative treatment for osteoid-osteoma. J Bone Joint Surg Am. 1992;74:179-85.

7. Kaweblum M, Lehman WB, Bash J, Grant AD, Strongwater A. Diagnosis of osteoid osteoma in the child. Orthop Rev. 1993;22:1305-13. 
8. Kaweblum M, Lehman WB, Bash J, Strongwater A, Grant AD. Osteoid osteoma under the age of five years. The difficulty of diagnosis. Clin Orthop Relat Res. 1993:218224.

9. Szabo RM, Smith B. Possible congenital osteoid-osteoma of a phalanx. A case report. J Bone Joint Surg Am. 1985;67:815-6.

10. Kransdorf MJ, Stull MA, Gilkey FW, Moser Jr RP. Osteoid osteoma. Radiographics. 1991;11:671-96.

11. Klein MH, Shankman S. Osteoid osteoma: radiologic and pathologic correlation. Skeletal Radiol. 1992;21:23-31.

12. Davies M, Cassar-Pullicino VN, Davies AM, McCall IW, Tyrrell PN. The diagnostic accuracy of MR imaging in osteoid osteoma. Skeletal Radiol. 2002;31:559-69.

13. Hayes CW, Conway WF, Sundaram M. Misleading aggressive MR imaging appearance of some benign musculoskeletal lesions. Radiographics. 1992;12:1119-34.

14. Byers PD. Solitary benign osteoblastic lesions of bone: osteoid osteoma and benign osteoblastoma. Cancer. 1968;22:43-57.

15. Norman A, Dorfman HD. Osteoid-osteoma inducing pronounced overgrowth and deformity of bone. Clin Orthop Relat Res. 1975;110:233-8.
16. Rosenthal DI, Alexander A, Rosenberg AE, Springfield D. Ablation of osteoid osteomas with a percutaneously placed electrode: a new procedure. Radiology. 1992;183:29-33.

17. Donkol RH, Al-Nammi A, Moghazi K. Efficacy of percutaneous radiofrequency ablation of osteoid osteoma in children. Pediatr Radiol. 2008;38:180-5.

18. Lindner NJ, Ozaki T, Roedl R, Gosheger G, Winkelmann W, Wortler K. Percutaneous radiofrequency ablation in osteoid osteoma. J Bone Joint Surg Br. 2001;83:391-6.

19. Cioni R, Armillotta N, Bargellini I, et al. CT-guided radiofrequency ablation of osteoid osteoma: long-term results. Eur Radiol. 2004;14:1203-8.

20. Ekstrom W, Soderlund V, Brosjo O. Osteoid osteoma in a 1-yearold boy - a case report. Acta Orthop. 2006;77:686-8.

21. Vanderschueren GM, Taminiau AH, Obermann WR, van den BergHuysmans AA, Bloem JL. Osteoid osteoma: factors for increased risk of unsuccessful thermal coagulation. Radiology. 2004;233:757-62.

22. Wortler K, Vestring T, Boettner F, Winkelmann W, Heindel W, Lindner N. Osteoid osteoma: CT-guided percutaneous radiofrequency ablation and follow-up in 47 patients. J Vasc Interv Radiol. 2001;12:717-22. 